\title{
WATER CONTENT, LENS HARDNESS AND CATARACT APPEARANCE
}

\author{
H. TABANDEH, G. M. THOMPSON, P. HEYWORTH, S. DOREY, A. J. WOODS and D. LYNCH \\ London
}

\begin{abstract}
SUMMARY
Hardening of a cataractous lens is associated with the clinical appearance of brunescence and advancing age. Alterations in the nature and concentration of proteins, lens fibre compaction and other biochemical changes may all be contributing factors towards increased hardness. As the nucleus confers most of the rigidity to the lens and water content decreases towards the centre of the nucleus, the hardness might be thought to be related to total water content. To study the relationship between water content, hardness and the clinical appearance of cataract, 135 lenses were obtained from eyes undergoing extracapsular cataract surgery. The cataracts were assessed and classified pre-operatively. Lens hardness was determined by a specially designed guillotine and water content was measured by weighing the lens before and after desiccation. A regression analysis was carried out to look at the variation of lens water content with hardness, degree of nuclear sclerosis, extent of cortical and posterior subcapsular cataract, vacuolation and age. Multivariate analysis of data demonstrated a relationship between lens hardness and lens water content, degree of nuclear sclerosis and age $\left(R^{2}=0.59\right)$. An association could not be detected between lens water content and age, degree of nuclear sclerosis, extent of cortical and posterior subcapsular cataract and vacuoles. This study suggests that hardening of the lens is reflected by a respective decrease in water content. Increased hardness is associated with coloration and advancing age.
\end{abstract}

With the advent of small-incision cataract surgery and phacoemulsification, assessment of 'hardness' of a cataractous lens has become relevant to daily clinical practice as this is a factor which influences the suitability of a patient for phacoemulsification. Lens hardness depends on factors such as changes in the nature of the lens proteins, compactness of fibres and other biochemical changes. A relationship between the clinical appearance of the cataract, particularly nuclear colour, and its hard-

Correspondence to: H. Tabandeh, Department of Ophthalmology, St George's Hospital, London SW17 OQT, UK. ness has been demonstrated.' There are few studies concerning the underlying biochemical changes that may be associated with increased hardness. One such factor is reduced water content.

This study aimed to investigate the possible association between increased hardness and relative dehydration of the crystalline lens. The relationship between degree of nuclear sclerosis, age and lens hardness was further studied.

\section{SUBJECTS AND METHODS}

One hundred and thirty-five patients with cataract underwent slit lamp examination prior to surgery in order to classify the type and degree of cataract. A classification system based on the Lens Opacities Classification System II was used.' Three main types of cataract were recognised, namely nuclear, posterior subcapsular and cortical. Each type of cataract was then compared with a standard set of colour photographs under constant illumination. The nuclear element of the cataract was evaluated by colour (range $0-4$ ) and by opalescence (range $0-4$ ). These two factors were assessed with the slit lamp beam at $45^{\circ}$ to the visual axis. Colours ranged from the faintest hint of yellow/green $(=1)$ to dark brown $(=4)$. Posterior subcapsular cataracts were assessed using retroillumination by the red reflex and a grading (0-3) was made according to the area of posterior pole involved by comparison with standardised colour photographs. Cortical cataracts were evaluated using retroillumination and comparing the area involved with the standardised colour photographs. In addition presence of vacuoles was graded against the red reflex $(0-3)$.

Following extracapsular extraction the sodium hyaluronate was cleared from the expressed nucleo-cortex and each lens was transferred to a moist chamber. Lens hardness was assessed by means of a specially devised automated lens guillotine which measured the maximum force required to bisect the lens. ' The wet weight was obtained by weighing both halves of the lens immediately after bisecting the lens using a microbalance (Metler AE 240). 
Table Ia. Age, percentage lens water content (IWC $\%$ ) and hardness (force)

\begin{tabular}{lcccccc}
\hline \multirow{2}{*}{$\begin{array}{l}\text { Age } \\
(\mathrm{yr})\end{array}$} & \multicolumn{2}{c}{ LWC $(\%)$} & & \multicolumn{2}{c}{ Force $(\mathrm{N})$} & \\
\cline { 2 - 3 } & Mean & SD & & Mean & SD & $F$ \\
\hline 60 & 63.8 & $(5.8)$ & & 0.76 & $(0.52)$ & 21 \\
$61-70$ & 66.0 & $(5.3)$ & & 0.93 & $(0.50)$ & 25 \\
$71-80$ & 64.2 & $(4.3)$ & & 1.10 & $(0.73)$ & 38 \\
$>80$ & 64.7 & $(4.2)$ & & 1.40 & $(0.56)$ & 49 \\
\hline
\end{tabular}

Table II. Nuclear colour, percentage lens water content (LWC\%), hardness (force) and age

\begin{tabular}{lccccccccc}
\hline & \multicolumn{2}{c}{ LWC $(\%)$} & & \multicolumn{2}{c}{ Force $(\mathrm{N})$} & & \multicolumn{2}{c}{ Age $(\mathrm{yr})$} & \\
\cline { 2 - 3 } Colour & Mean & SD & & Mean & SD & & Mean & SD & $F$ \\
\hline 1 & 64.9 & $(6.1)$ & & 0.59 & $(0.35)$ & & 71.1 & $(13.7)$ & 36 \\
2 & 64.1 & $(4.8)$ & & 0.97 & $(0.45)$ & & 74.0 & $(13.9)$ & 34 \\
3 & 64.7 & $(3.8)$ & & 1.38 & $(0.48)$ & & 74.7 & $(10.2)$ & 41 \\
4 & 65.3 & $(3.4)$ & & 1.82 & $(0.66)$ & & 76.5 & $(9.1)$ & 24 \\
\hline
\end{tabular}

Table IV. Extent of posterior subcapsular lens opacity (PSCLO), nuclear colour, percentage lens water content (IWC $\%$ ). hardness (force) and age

\begin{tabular}{lcclllllll}
\hline & \multicolumn{2}{c}{ LWC $(\%)$} & & \multicolumn{2}{c}{ Force $(\mathrm{N})$} & & \multicolumn{2}{c}{ Age $(\mathrm{yr})$} & \\
\cline { 2 - 3 } PSCLO & Mean & SD & & Mean & SD & & Mean & SD & $F$ \\
\hline 0 & 65.2 & $(3.5)$ & & 1.68 & $(0.61)$ & & 76.6 & $(7.9)$ & 21 \\
1 & 65.0 & $(4.2)$ & & 1.20 & $(0.63)$ & & 74.6 & $(10.9)$ & 32 \\
2 & 64.2 & $(5.6)$ & & 0.99 & $(0.55)$ & & 73.4 & $(15.2)$ & 47 \\
3 & 64.4 & $(3.9)$ & & 0.88 & $(0.53)$ & & 72.3 & $(10.4)$ & 32 \\
\hline
\end{tabular}

The lens was then placed in an oven $\left(80{ }^{\circ} \mathrm{C}\right)$ containing desiccant silica gel and was re-weighed after 24 hours and again at regular intervals until a constant weight was achieved (to the nearest $0.1 \mathrm{mg}$ ). This was taken as the dry weight and percentage water content was calculated as: [(wet weight - dry weight $) /$ wet weight $] \times 100$.

\section{Statistical Methods}

The univariate relationship between lens water content and lens hardness, nuclear sclerosis, age group, extent of cortical and posterior cataracts and vacuoles was examined using one-way analysis of variance except where variances were found to be non-homogeneous, when Kruskal-Wallis tests were used. Variances were examined using Bartlett's test. Chi-squared tests were used for comparisons between pairs of discrete variables except where expected cell values were less than 5, when Fisher's exact two-tailed test was used.

The relationships and their interactions were examined in a multivariate analysis. The sample size was 130 subjects for multivariate analysis due to 5 subjects having some missing data. The statistical analysis package SAS was used to analyse the data and multivariate regression was performed by generalised linear modelling (GLM).

\section{RESULTS}

A total of 135 lenses were studied. Eighty-five cataracts were thought to be senile in origin, 26 were associated
Table Ib. Age, hardness (force) and type of cataract in lenses with extreme values of water content (LWC\%)

\begin{tabular}{lcccccc}
\hline LWC (\%) & Age $(\mathrm{yr})$ & Force $(\mathrm{N})$ & Colour & Cortex & PSCLO & Vacuoles \\
\hline 79 & 62 & 0.18 & 1 & 0 & 1 & 2 \\
78 & 29 & 0.16 & 1 & 3 & 2 & 1 \\
76 & 88 & 1.2 & 2 & 3 & 2 & 1 \\
46 & 82 & 1.6 & 1 & 2 & 2 & 0 \\
\hline
\end{tabular}

PSCLO, posterior subcapsular lens opacity.

Table III. Extent of cortical lens opacity (CLO), percentage lens water content (LWC\%), hardness (force) and age

\begin{tabular}{lccccccccc}
\hline & \multicolumn{2}{c}{} & \multicolumn{2}{c}{ IWC $(\%)$} & & \multicolumn{2}{c}{ Force $(\mathrm{N})$} & & \multicolumn{2}{c}{ Age $(\mathrm{yr})$} & \\
\cline { 2 - 3 } CLO & Mean & SD & & Mean & SD & & Mean & SD & $F$ \\
\hline 0 & 65.4 & $(4.3)$ & & 1.23 & $(0.77)$ & & 71.1 & $(10.2)$ & 32 \\
1 & 63.3 & $(4.2)$ & & 1.22 & $(0.55)$ & & 71.1 & $(12.9)$ & 50 \\
2 & 65.8 & $(5.1)$ & & 0.94 & $(0.55)$ & & 74.0 & $(8.3)$ & 25 \\
3 & 65.2 & $(6.3)$ & & 0.86 & $(0.55)$ & & 74.7 & $(9.0)$ & 12 \\
4 & 65.2 & $(4.7)$ & & 0.93 & $(0.67)$ & & 76.5 & $(10.2)$ & 14 \\
\hline
\end{tabular}

Table V. Extent of vacuole formation, percentage lens water content (LWC\%), hardness (force) and age

\begin{tabular}{|c|c|c|c|c|c|c|c|}
\hline \multirow[b]{2}{*}{ Vacuole } & \multicolumn{2}{|c|}{$\operatorname{LWC}(\%)$} & \multicolumn{2}{|c|}{ Force $(\mathrm{N})$} & \multicolumn{2}{|c|}{ Age (yr) } & \multirow[b]{2}{*}{$F$} \\
\hline & Mean & SD & Mean & SD & Mean & SD & \\
\hline 0 & 64.0 & $(4.91)$ & 1.28 & $(0.74)$ & 73.6 & (11.7) & 50 \\
\hline 1 & 65.1 & $(4.4)$ & 1.04 & $(0.51)$ & 74.4 & $(12.9)$ & 62 \\
\hline 2 & 65.3 & $(5.2)$ & 0.90 & $(0.64)$ & 71.4 & (12.2) & 20 \\
\hline 3 & 60.4 & $(-)$ & 1.00 & $(-)$ & 76.0 & $(-)$ & 1 \\
\hline
\end{tabular}

with diabetes and 13 had other cataract risk factors such as chronic use of systemic steroids, retinitis pigmentosa, Down's syndrome and congenital lens opacities. In the young patient group (age $\leqslant 60$ years), 10 cataracts were associated with risk factors such as diabetes mellitus, strong family history or chronic ocular disease, and in 11 subjects there were no identifiable risk factors. The mean age was 73.6 years with a range of $29-102$ years. The average force required to bisect the lenses was $1.12 \mathrm{~N}$. A twentyfold difference was present between the softest and the hardest lenses. The mean water content was $64.7 \%$.

\section{Lens Hydration and Hardness}

Lens hydration and hardness are significantly correlated (Pearson's correlation coefficient $=-0.2$, significant at the $5 \%$ level, confidence limits $-0.37<R<-0.05)$. The lens hardness increases as the water content falls (Fig. 1).

Age

There is no significant difference in mean lens water content (LWC) between various age groups (Table Ia, Fig. 2). The extreme LWC values of $79 \%, 78 \%, 76 \%$ and $46 \%$ had ages of $62,29,88$ and 82 years respectively (Table Ib). A significant difference in mean force is observed between age groups $(F=5.9, p=0.0012)$ (Fig. 3).

\section{Nuclear Sclerosis}

Mean LWC did not vary significantly with increasing col- 


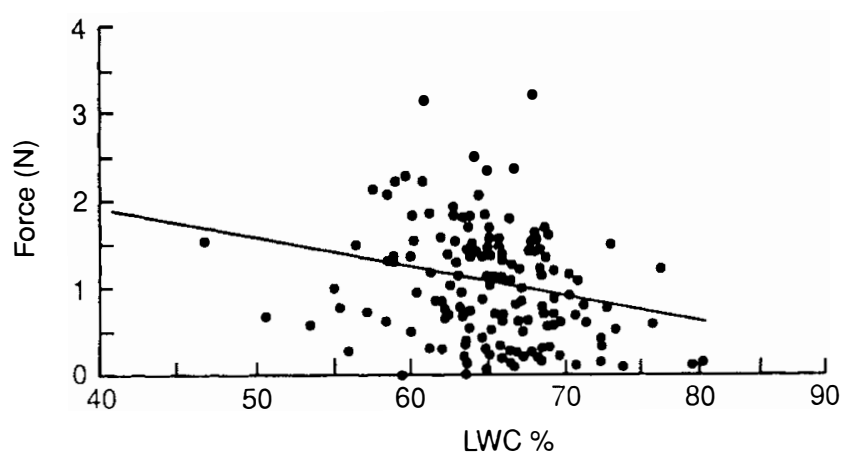

Fig. 1. Lens hardness (force) in relation to lens water content $(L W C \%)$.

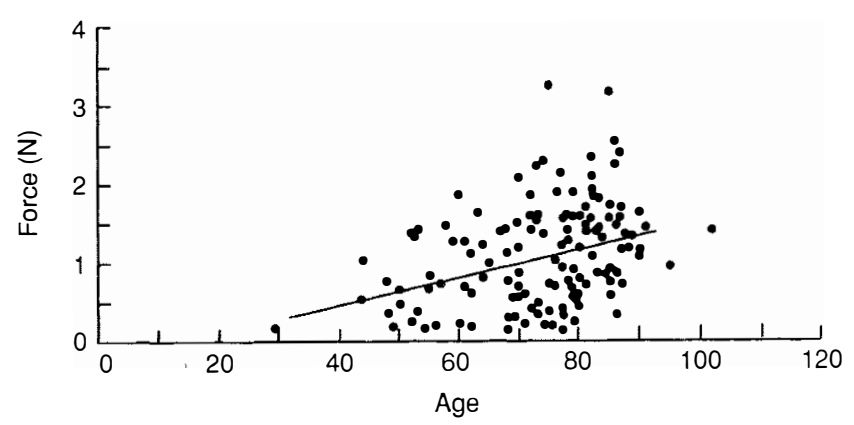

Fig. 3. Lens hardness (force) in relation to age (yr).

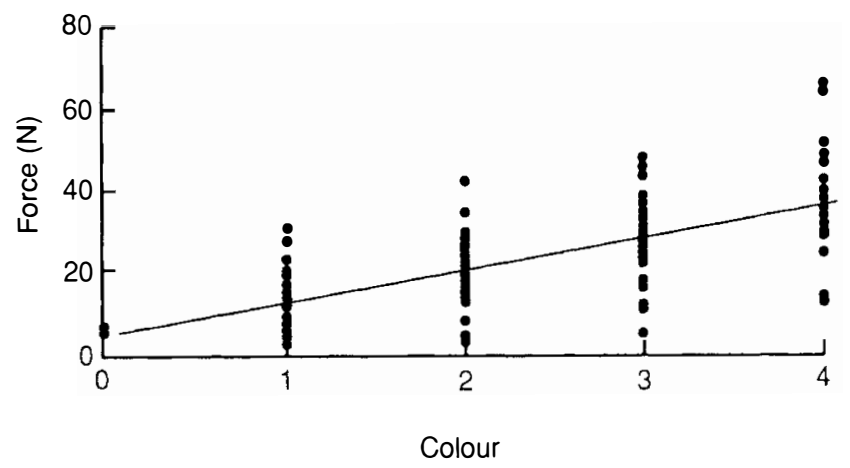

Fig. 5. Lens hardness (force) in relation to muclear colour.

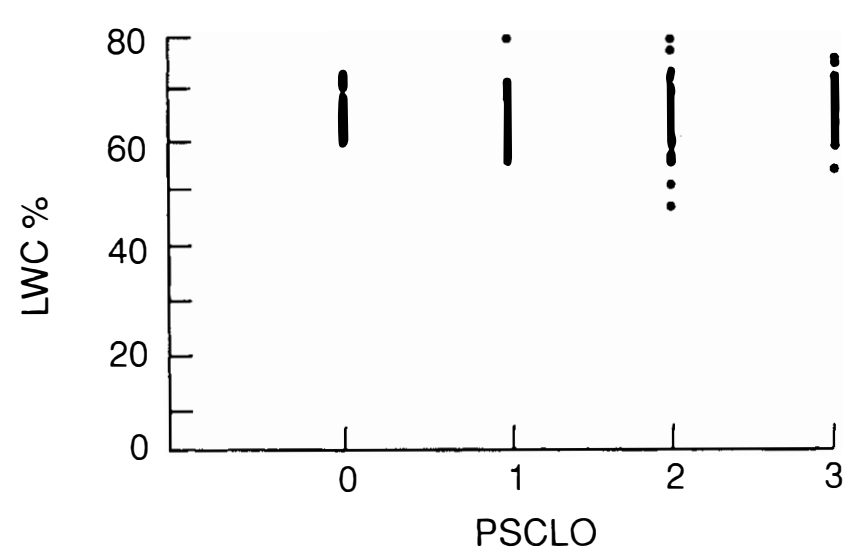

Fig. 7. Lens water content $(L W C \%)$ in relation to the extent of posterior subcapsular lens opacity (PSCLO).

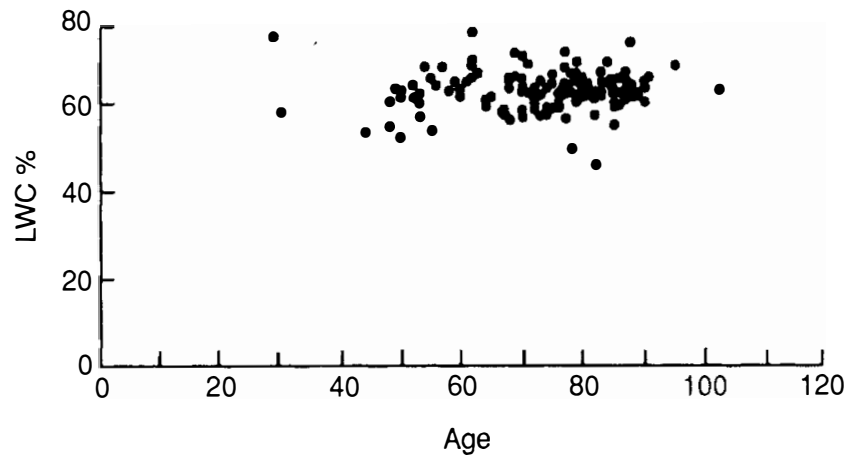

Fig. 2. Lens water content (LWC\%) in relation to age (w).

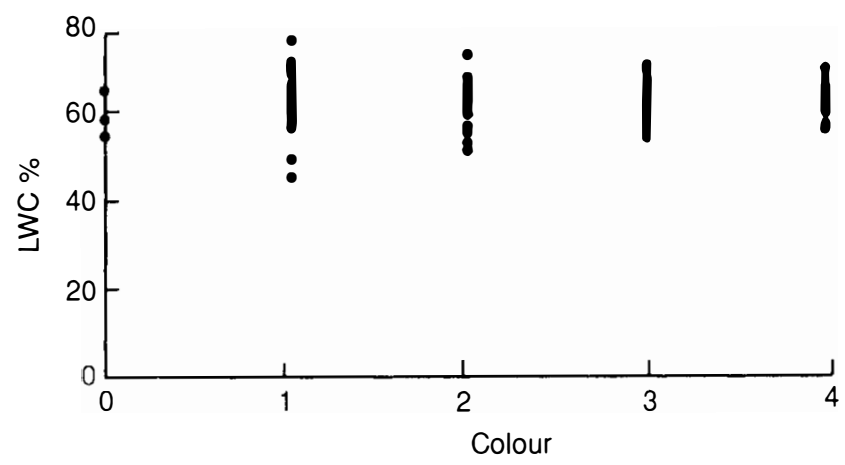

Fig. 4. Lens water content (LWC\%) in relation to nuclear colour.

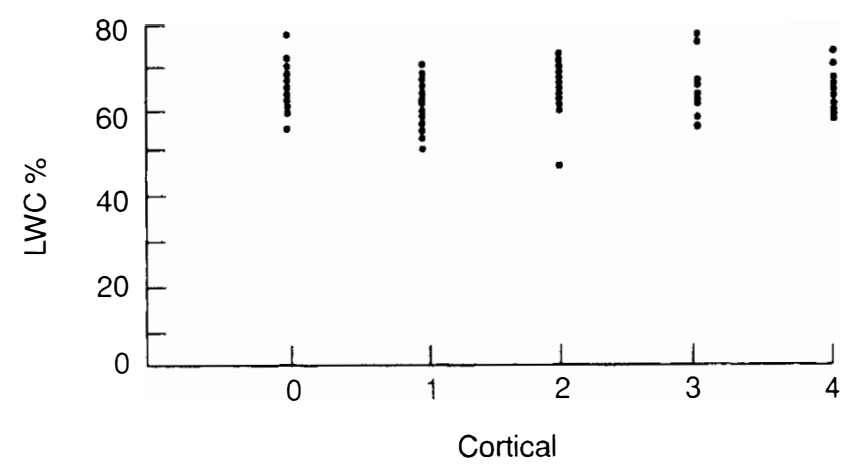

Fig. 6. Lens water content ( $L W(\%)$ in relation to the extent of cortical lens opacity. 
oration (Table II, Fig. 4). There is a significant relationship between colour and hardness (Kruskal-Wallis 61.9, d.f. $=3, p<0.000001)($ Table II, Fig. 5).

\section{Cortical Cataracts, Posterior Subcapsular Cataracts and Vacuoles}

Hydration and hardness are not significantly related to the degree of cortical cataract, posterior subcapsular cataract or presence of vacuoles (Table III-V, Figs. 6 and 7).

Using lens hardness (force) as the dependent variable, lens water content (LWC), lens colour and age were found to be important explanatory variables. The following model explained $59 \%$ of the variation in lens hardness:

Lens hardness $($ force $)=3.1-(0.04 \times \mathrm{LWC})+(0.1 \times$ Age $)-(1.2 \times$ Colour 1$)$ $-(0.9 \times$ Colour 2$)$ $-(0.5 \times$ Colour 3$)$

For the overall model $F=36.2$, degrees of freedom $=5,129, p<0.0001$; and for the individual parameters of colour, age and lens water content $F=46.8$, 20.3 and 20.2 respectively, and all $p$ values $<0.0001$. There are variables not measured in this study which must be contributing significantly to lens hardness. However, the major parameter in explaining the hardness is the colour of the lens. A model for the natural log of lens hardness and water content was tried but did not improve the model significantly.

\section{DISCUSSION}

As small-incision cataract surgery and phacoemulsification become increasingly popular, hardness of the lens has also become clinically relevant. Hardness of the crystalline lens is a physical property which has not been the subject of much investigation in the past. This study set out to identify a possible association between increased hardness and relative dehydration of the crystalline lens.

Lens hardness is dependent upon factors such as changes in the nature of the lens proteins and compactness of fibres. In the nucleus, where the lens becomes harder, the solubility of crystalline proteins is reduced concomitantly with the protein thiols becoming oxidised. Some amino acids, especially aromatics and methionine sulphurs, make particularly good 'glue' for pasting together protein units. ${ }^{3}$ Further evidence for the effect of increased insoluble proteins upon hardness is provided by the continuous cortex-to-core increase in this protein fraction around the age of onset of presbyopia, which is known to be associated with increased lens rigidity. ${ }^{4}$ These alterations in protein structure, in particular those that alter their potential for intermolecular interactions, result in macroscopic effects such as lens colour changes as well as an increase in lens rigidity.

\section{Lens Hydration and Hardness}

Although some researchers have stated that dehydration of human lens is of little importance, in theory relative dehydration of the lens may be associated with increased con- centration of water-insoluble proteins and also further compaction of the lens fibres thereby increasing hardness. Studies involving microsectioning techniques suggest that dehydration and consequently the increase in dry weight and water-insoluble fraction occur progressively towards the centre of the nucleus. ${ }^{5}$ It is also established that the nucleus confers most of the rigidity and hardness to the lens. This study finds a relative dehydration of the lens in association with increasing hardness. Water in the crystalline lens may be in a free state or be bound to the protein molecules. It is likely that the relative dehydration demonstrated by this study is a reflection of loss of both protein bound and free water within the nucleus. Reduced water content is probably associated with changes in the interaction between protein chains as well as increased protein concentration resulting in hardness. There is a wide spread of values for force and water content, indicating that there are variables not measured in this study which must be contributing to lens hardness.

\section{Age}

This study, whilst confirming a relationship between ageing and lens hardness, does not find a significant association between ageing and water content. In other reported studies the relationship between ageing and lens water content is not clear. In animal lenses the water content is highest at birth and decreases during adolescence. ${ }^{6.7}$ While some studies in human adults suggest a relative dehydration of the lens with age, others have not found a significant relationship.

\section{Nuclear Sclerosis}

Nuclear colour is taken as representing sclerosis. The precise nature of the yellow coloration of the lens is unknown. However, it correlates well with many biochemical changes. Colour appears to be mostly associated with an increase in water-insoluble lens proteins and in particular the urea-insoluble subfraction. ${ }^{10-13}$ It has been proposed that substances derived from tyrosine and tryptophan by photo-oxidation produce an insoluble pigmented moiety. With continued alteration of the proteins in nuclear cataract, the coloration becomes more intense. This study does not demonstrate a relationship between the degree of nuclear sclerosis and lens hydration. The clinical impression that brunescence is associated with hardening of the lens has previously been demonstrated by measuring hardness in nuclear cataracts of various grading. This study further confirms this relationship.

\section{Cortical Cataracts, Posterior Subcapsular Cataracts and Vacuoles}

Deussen and Pau reported a higher ratio of cortical to nuclear water content in cortical, particularly posterior subcapsular, cataracts than in other types of cataract. The same cataracts also exhibited a lower total lens weight due to loss of proteins. ${ }^{1+}$ Other studies suggest increased total lens hydration in cortical cataracts. ${ }^{15}$ In this study there is no significant difference in the total lens water content 
between grade 4 cortical, grade 3 posterior subcapsular and grade 4 nuclear cataracts. In addition the extent of various types of cataracts did not influence total water content or lens hardness.

Vacuoles are thought to be water-filled spaces within and without the lens fibres. They are traditionally considered to be associated with an increase in lens hydration as well as being an indicator of a looser arrangement of lens fibres and therefore softness. However, a relationship between the extent of vacuole formation and lens hydration or hardness could not be demonstrated. Therefore the clinical expression that vacuole formation represents a softer lens is not justified.

\section{CONCLUSION}

In summary, with the advent of phacoemulsification lens hardness has become clinically relevant. It is also likely that hardness will be of relevance to future methods of cataract surgery. This study demonstrates that hardening of the lens nucleus is associated with coloration and advancing age. Whilst there does not appear to be a relationship between hydration and nuclear coloration, increased hardness is reflected by a respective decrease in water content. Factors influencing hardening of the human lens are not clear and further research is warranted in order to establish the biochemical changes that influence hardness. Identification of factors which may reverse this process will be of therapeutic value in terms of both prevention and surgical treatment of cataract.

The authors wish to thank Dr D. K. Nassiri. Mr Peter Holland, Dr Peter Ayliffe, Mr Don Ritchie, Mr George Gibson and Mr Ian Eversden for their technical support.

Key words: Cataract classification, Lens hardness. Nuclear sclerosis. Phacoemulsification, Water content.

\section{REFERENCES}

1. Heyworth P. Thompson GM, Tabandeh H, McGuigan S. Relationship between clinical classification of cataract and its hardness. Eye 1993:7:726-30.

2. Chylack LT, Leske MC, McCarthy D, Khu P, Kashiwagi T, Sperduto R. Lens Opacities Classification System II (LOCS II). Arch Ophthalmol 1989:107:991-7.

3. Argos P. An investigation of protein subunit and domain interfaces. Protein Eng 1988;2:101-13.

4. Li LK. Roy D, Spector A. Changes in lens protein in concentric fractions from individual normal human lenses. Curr Eye Res 1986;5:127-35.

5. Fisher RF, Pettet BE. Presbyopia and the water content of the human crystalline lens. J Physiol (Lond) 1973;234:443-7.

6 . Fisher RF. The changes with age in the biophysical properties of the capsula of the human crystalline lens in relation to cataract. Interdiscipl Top Gerontol 1978;12:131-43.

7. Rink H. The water content of bovine lenses during aging. Interdiscipl Top Gerontol 1978;12:271-7.

8. Hockwin O, Rast F, Rink H. Water content of lenses of different species. Interdiscipl Top Gerontol 1978;13:102-8.

9. Bours J, Fodisch H, Hockwin O. Age-related changes in water and crystalline content of the fetal and adult human lens, demonstrated by a microsectioning technique. Ophthalmic Res 1987:19:235-9.

10. Pirie A. Colour and solubility of the proteins of human cataracts. Invest Ophthalmol 1968;7:634-50.

11. Van Heyningen R. The human lens. I. A comparison of cataracts extracted in Oxford (England) and Shikarpur (W. Pakistan). Exp Eye Res 1972:13:136-47.

12. Van Heyningen R. The human lens. III. Some observations in the post-mortem lens. Exp Eye Res 1972;13:155-60.

13. Zigman S. Eye lens colour: formation and function. Science 1971:171:807-9.

14. Deussen A, Pau H. Regional water content of clear and cataractous human lenses. Ophthalmic Res 1989;21:374-80.

15. Pope JM, Chandra S, Balfe JD. Changes in the state of water in senile cataractous lenses as studied by nuclear magnetic resonance. Exp Eye Res 1982:34:57-63. 